International Mathematical Forum, Vol. 8, 2013, no. 18, 859 - 863 HIKARI Ltd, www.m-hikari.com

\title{
On a Class of Nowhere Commutative Semigroup Rings
}

\author{
B. Ahmadi \\ Department of Mathematics, Science and Research Branch \\ Islamic Azad University \\ P.O. Box 14515/775, Tehran, Iran \\ b.ahmadi1358@yahoo.com
}

\section{H. Doostie}

Department of Mathematics, Science and Research Branch Islamic Azad University

P.O. Box 14515/775, Tehran, Iran

doostih@gmail.com

Copyright (C) 2013 B. Ahmadi and H. Doostie. This is an open access article distributed under the Creative Commons Attribution License, which permits unrestricted use, distribution, and reproduction in any medium, provided the original work is properly cited.

\begin{abstract}
For a finite algebraic structure $A$ the commutativity degree of $A$, denoted by $P_{r}(A)$, is the probability that two elements of $A$ commute. Since 1973, $P_{r}(A)$ studied for finite groups showing that $P_{r}(A) \leq \frac{5}{8}$. The study of "almost commutative" semigroups in 2011 showed that for some finite semigroup $A, P_{r}(A)>\frac{5}{8}$ and even $P_{r}(A)$ may be arbitrarily close to 1 . Since $0 \leq P_{r}(A) \leq 1$ then looking for algebraic structures $A$ such that $P_{r}(A) \rightarrow 0$, is of interest, for, the centralizer of every element of such $A$ should be a singleton. In this paper for every integer $n \geq 2$ and every prime $p \geq 3$ we give an infinite class of finite semigroup rings $A_{n, p}=Z_{p}\left(P_{n}\right)$ of order $p^{n}$ where, $P_{n}$ is a non-commutative semigroup and show that $P_{r}\left(A_{n, p}\right) \rightarrow 0$, for sufficiently large values of $n$ and $p$. We name such semigroup rings as "extremely non-commutative" semigroup rings.
\end{abstract}

Mathematics Subject Classification: 20M05, 20M25 
Keywords: Semigroup rings, commutativity degree, presentation of semigroups

\section{Introduction}

For a given finite algebraic structure $A$ (group, ring, semigroup or semigroup ring), the commutativity degree of $A$ is defined to be

$$
P_{r}(A)=\frac{\left|\left\{(x, y) \in A^{2} \mid x y=y x\right\}\right|}{\left|A^{2}\right|} .
$$

The study of this probability for finite groups started in 1973 by Gustafson [5] by showing that $P_{r}(A) \leq \frac{5}{8}$ for every non-abelian group $A$. The commutativity for finite rings defined and studied by McHale in 1976 (see [8]). The article [7] studied the case when $P_{r}(A) \geq \frac{1}{2}$ in 1995 for a finite group $A$. In the article [4] the case $P_{r}(A)<\frac{1}{2}$ and specially when $\frac{1}{4}<P_{r}(A)<\frac{1}{2}$ studied in 2008 by giving certain infinite classes of finite groups. One of the three studied classes in this article satisfies the condition $P_{r}(A) \rightarrow 0$ for the sufficiently large group A.

The study of this probability for semigroups started in 2011 by Ahmadidelir

[1] where the authors proved that for some semigroup $A, P_{r}(A)>\frac{5}{8}$. The natural question that "for which algebraic structure $A$ (except the groups), $P_{r}(A) \rightarrow 0$ " may be posed here and in what follows we construct a class of two parametric semigroup rings $A_{n, p}$ of order $p^{n}$, for every integer $n \geq 2$ and odd prime $p$, to show that $P_{r}\left(A_{n, p}\right) \rightarrow 0$. We name such an algebraic structure the "extremely non-commutative" algebraic structure.

Our notation is fairly standard and follows $[2,3,6,9]$ on the presentations of groups and semigroups. We may also recall the definition of a group $\operatorname{ring} R[S]$ for a commutative ring $R$ and an arbitrary finite semigroup $S$, consisting of all linear combinations $\sum_{g \in S} \alpha(g) g$, where $\alpha(g) \in R$ and $\alpha(g)=0$ except for a finite number of coefficients. Of course the sum and multiplication of elements in $R[S]$ are defined as usual by:

$$
\begin{gathered}
\left(\sum_{g \in S} \alpha(g) g\right)+\left(\sum_{g \in S} \beta(g) g\right)=\sum_{g \in S}(\alpha(g)+\beta(g)) g \\
\left(\sum_{g \in S} \alpha(g) g\right)\left(\sum_{h \in S} \beta(h) h\right)=\sum_{g, h \in S}(\alpha(g) \beta(h)) g h .
\end{gathered}
$$

Let $n \geq 2$ be an integer and $p$ an odd prime. Consider the presentation

$$
\pi=\left\langle a_{1}, a_{2}, \ldots, a_{n} \mid a_{i}^{2}=a_{i}, a_{i+1} a_{i}=a_{i+1}, a_{i} a_{i+1}=a_{i},(1 \leq i \leq n)\right\rangle
$$

where, the indices are reduced modulo $n$. The semigroup defined by $\pi$ (indeed, $S g(\pi)$, as a usual notation of the finitely presented structures), will be dented 
by $P_{n}$. Finally, by denoting the semigroup $\operatorname{ring} Z_{p}\left[P_{n}\right]$ by $A_{n, p}$ we give our main results as:

Proposition 1.1 The semigroup ring $A_{n, p}$ is finite of order $p^{n}$. Moreover, for every integer $n \geq 2$, the inequalities $\frac{1}{2 p^{2}} \leq P_{r}\left(A_{n, p}\right) \leq \frac{1}{p^{2}}$ hold, for every odd prime $p$.

Corollary 1.2 The semigroup ring $A_{n, p}$ is extremely non-commutative.

\section{Preliminary results}

Every element of $A_{n, p}$ is in the form $x=\alpha_{1} a_{1}+\alpha_{2} a_{2}+\ldots+\alpha_{n} a_{n}$. For a given values of $\alpha_{1}, \alpha_{2}, \ldots, \alpha_{n-1}$ define the set $A\left(\alpha_{1}, \alpha_{2}, \ldots, \alpha_{n-1}\right)$ as follows:

$$
A\left(\alpha_{1}, \alpha_{2}, \ldots, \alpha_{n-1}\right)=\left\{\left(\alpha_{1}, \alpha_{2}, \ldots, \alpha_{n-1}, \alpha_{i}\right) \mid \alpha_{i} \in Z_{p}\right\}
$$

Then, as a preliminary result we get:

Lemma 2.1 For every $\alpha_{1}, \alpha_{2}, \ldots, \alpha_{n-1}$, the set $A\left(\alpha_{1}, \alpha_{2}, \ldots, \alpha_{n-1}\right)$ contains exactly one $n$-typle $\left(\alpha_{1}, \alpha_{2}, \ldots, \alpha_{n-1}, \beta\right)$ satisfying $\beta+\sum_{i=1}^{n-1} \alpha_{i} \equiv 0(\bmod p)$, where, $\beta \in Z_{p}$.

Proof For two different elements $\left(\alpha_{1}, \alpha_{2}, \ldots, \alpha_{n-1}, \beta\right)$ and $\left(\alpha_{1}, \alpha_{2}, \ldots, \alpha_{n-1}, \gamma\right)$ of $A\left(\alpha_{1}, \alpha_{2}, \ldots, \alpha_{n-1}\right)$ we easily get $\beta-\gamma \equiv 0(\bmod p)$ which gives the contra$\operatorname{diction} \beta=\gamma$.

Lemma 2.2 The semigroup ring $A_{n, p}$ contains exactly $p^{n-1}$ elements satisfying $\sum_{i=1}^{n} \alpha_{i} \equiv 0(\bmod p)$.

Proof There are exactly $p^{n-1}$ sets as $A\left(\alpha_{1}, \alpha_{2}, \ldots, \alpha_{n-1}\right)$. On the other hand, there are a one-one correspondence between the elements of the set $A_{n, p}$ and the set

$$
\bigcup_{\left(\alpha_{1}, \alpha_{2}, \ldots, \alpha_{n-1}\right) \in Z_{p}^{n-1}} A\left(\alpha_{1}, \alpha_{2}, \ldots, \alpha_{n-1}\right) .
$$

So, the result follows at once by using the Lemma 2.1 .

Lemma 2.3 Two different elements $x=\alpha_{1} a_{1}+\alpha_{2} a_{2}+\ldots+\alpha_{n} a_{n}$ and $y=$ $\beta_{1} a_{1}+\beta_{2} a_{2}+\ldots+\beta_{n} a_{n}$ of $A_{n, p}$ commute if and only if $\sum_{i=1}^{n} \alpha_{i} \equiv 0(\bmod p)$ and $\sum_{i=1}^{n} \beta_{i} \equiv 0(\bmod p)$. 
Proof Considering the relators of the semigroup $P_{n}$ we get:

$$
\begin{aligned}
& x y=y x \Leftrightarrow \sum_{i=1}^{n} \alpha_{i}\left(\beta_{1}+\beta_{2}+\ldots+\beta_{n}\right) a_{i}=\sum_{i=1}^{n} \beta_{i}\left(\alpha_{1}+\alpha_{2}+\ldots+\alpha_{n}\right) a_{i} \\
& x y=y x \Leftrightarrow\left(\beta_{1}+\beta_{2}+\ldots+\beta_{n}\right) x=\left(\alpha_{1}+\alpha_{2}+\ldots+\alpha_{n}\right) y .
\end{aligned}
$$

Consequently, the last equation holds if and only if $\sum_{i=1}^{n} \alpha_{i} \equiv 0(\bmod p)$ and $\sum_{i=1}^{n} \beta_{i} \equiv 0(\bmod p)$, for, $x \neq y$.

\section{Results and Discussion}

By using the results of Section 2 we give here the proofs of Proposition 1.1 and corollary 1.2.

Proof of Proposition 1.1 The first part is obvious because of the representation of the elements of $A_{n, p}$. For the second part we have to compute $P_{r}\left(A_{n, p}\right)$, for a given $n$ and $p$. By the definition we get that

$$
P_{r}\left(A_{n, p}\right)=\frac{\left|A_{n, p}\right|+\left|\left\{(x, y) \in A_{n, p}^{2} \mid x \neq y, x y=y x\right\}\right|}{\left|A_{n, p}^{2}\right|} .
$$

Considering the Lemma 2.3 gives us the cardinal of the set

$$
\left\{(x, y) \in A_{n, p}^{2} \mid x \neq y, x y=y x\right\}
$$

as $\frac{p^{n-1}\left(p^{n-1}-1\right)}{2}$. So,

$$
P_{r}\left(A_{n, p}\right)=\frac{2 p^{n}+p^{n-1}\left(p^{n-1}-1\right)}{2 p^{2 n}}
$$

Or simply,

$$
P_{r}\left(A_{n, p}\right)=\frac{2 p+p^{n-1}-1}{2 p^{n+1}} .
$$

Consequently, for a given value of $n$, we may check the inequalities

$$
\frac{1}{2 p^{2}} \leq \frac{2 p+p^{n-1}-1}{2 p^{n+1}} \leq \frac{1}{p^{2}}
$$

for every prime $p \geq 3$.

Proof of Corollary 1.2 Since $\lim _{n \rightarrow \infty} \frac{2 p+p^{n-1}-1}{2 p^{n+1}}=\frac{1}{p^{2}}$ then, for the sufficiently large values of $p, P_{r}\left(A_{n, p}\right) \rightarrow 0$ showing that $A_{n, p}$ is an extremely non-commutative semigroup ring. 


\section{References}

[1] K. Ahmadidelir, C.M. Campbell and H. Doostie, Almost commutative semigroups, Algebra Colloquium, 18 (2011), 881-888.

[2] C.M. Campbell, E.F. Robertson, N. Ruskuc and R.M. Thomas, Semigroup and group presentations, Bull. London Math Soc. ,27 (1995), 46-50.

[3] C.M. Campbell, E.F. Robertson, N. Ruskuc, R.M. Thomas and Y. Unlu, Certain one relator products of semigroups, Comm. Algebra,23 (14) (1995), 5207-5219.

[4] H. Doostie and M. Maghasedi, Certain classess of groups with commutativity degree $d(G)<\frac{1}{2}$, Ars Combinatoria, 89 (2008), 263-270.

[5] W.H. Gustafson, What is the probability that two group elements commute, Amer. Math. Monthly,80 (1973), 1031-1034.

[6] D.L. Johnson, Presentation of groups, Cambridge University Press, Cambridge, 1997.

[7] P. Lescot, Isoclinism classes and commutativity degree of finite groups, J. Algebra, 177 (1995), 847-869.

[8] D. MacHale, Commutativity in finite rings, Amer. Math. Monthly,83 (1976), 30-32.

[9] E.F. Robertson and Y. Unlu, On semigroup presentations, Proc. Edinburgh Math. Soc. ,36 (1993), 55-68.

Received: September 19, 2012 